\title{
Automatic Marching Cubes for Improving 3D Medical Images Reconstruction
}

Farah Fekraoui ${ }^{1}$, Mohamed Chaouki Babahenini ${ }^{2}$

1,2 LISIA laboratory, Department of Computer Science

Biskra University, Biskra, Algeria

\{farah_fek@yahoo.fr\}\{chaouki.babahenini@gmail.com\}

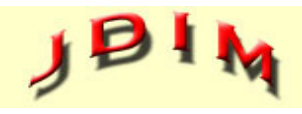

Journal of Digital Information Management
ABSTRACT: The Marching Cubes algorithm is based on the estimate of the isovalue in order to determine all the pixels that are belonging to the volume to be reconstructed. This estimation is done by the user in an interactive and way without any orientation; this is no longer an intuitive process. Solutions are proposed to detect more than one isovalue and have been used to display multiple isosurfaces and not to adjust or automatically determine isovalue. Other solutions propose to explore histogram to estimate isovalue automatically; those remain insufficient because they still require user intervention. The proposed method acts directly in the isovalue estimation phase. In fact, we propose to make this estimate by using automatic methods of sampling, which have shown their performance in estimating a threshold in several works. This paper proposes to make the use of existing automated isovalue selection methods that take into account histogram and the dynamics of the image. The automatic Marching Cubes algorithm reduces user interaction and makes the selection process more intuitive. The obtained results will show that this adaptation minimizes the computing time and that the obtained volumes are of better quality than those obtained by the classical Marching Cubes algorithm.

Subject Categories and Descriptors:

[I.3.3 Picture/Image Generation]; [I.4.5 Image Reconstruction]; [J.3 LIFE AND MEDICAL SCIENCES]; Medical information systems

General Terms: Medical Images, Image Processing, 3D Images, Marching Cubes Algorithms

Keywords: 3D Reconstruction, Marching Cubes Algorithm, Automatic Marching Cubes, Automatic Isovalue Selection,
Medical Image, Otsu’s Algorithm

Received: 18 August 2019, Revised 20 October 2019, Accepted 30 November 2019

Review Metrics: Review Scale: 0-6, Review Score: 4.95, Interreviewer consistency: $90.45 \%$

DOI: $10.6025 / \mathrm{jdim} / 2020 / 18 / 1 / 1-10$

\section{Introduction}

With the progress and evolution of the number of medical data, medical experts and scientists are using 3D visualization to improve and accelerate the analysis data task. The 3D reconstruction algorithm of Marching Cubes is currently the most popular algorithm for isosuface extraction.

The Marching Cubes is a 3D reconstruction algorithm from 2D images proposed by Lorensen and Clay (William and Harvey, 1987); it is currently one of the most popular algorithms for isosurface extraction. Since its appearance, this algorithm is widely used mainly in the medical field, since it allows obtaining very good approximations of the original surfaces from serial sections. Moreover, it is simple, fast and robust (Newman and Yi, 2006).

The marching cubes algorithm works on the divided and conquers principle. The volumetric dataset is divided into cells and the isosurface within each cell is calculated by thresholding volume data based on an isovalue. Determining an appropriate isovalue is a challenge inherent in any isosurface extraction method. Marching Cubes algorithm remains the choice of an appropriate isovalue to the user. 
The interactive estimation without any orientation or constraint of the isovalue is not optimal and it makes the Marching cubes algorithm supervised; that is to say piloted by the user. This operation can be expensive in computing time during multiple tests and it provides some construction's error. These tedious trial-and-error processes led to the desire to have them replaced by an automated method.

Therefore, we propose in this paper to automate the Marching Cubes algorithm, so that it is more independent of the user and more accurate while minimizing the computing time (saving the user from using multiple tests).

The remainder of the paper is organized as follows: Section 2 presents related works, Section 3 describes the proposed approach along with a brief explanation of the Marching Cubes Algorithm, and Section 4 discusses experimental results. Finally, Section 5 summarizes the paper and makes concluding remarks.

\section{Related Works}

One of the problems of the Marching Cubes algorithm is the selection of isovalue. Have yet to be solved satisfactorily. If the desired isosurface's isovalue is known in advance, the algorithm can be run once with an acceptable delay between value selection and visible output. However, when the isovalue is unknown and the viewer wishes to explore the data, this delay makes interaction difficult, especially as the volume grows in size (Lyness et Blake, 2001).

Many extensions and improvements have been made to the original Marching Cubes algorithm to including the solution of the interactive estimation of the isovalue. For the extraction of isosurfaces, the user interaction for the selection of an appropriate isovalue is no longer an intuitive process. J. Marks and authors (Marks et al., 1997) replace the trial-and-error process of producing and displaying a high number of results using various possible transfer functions, which reduce user interaction and make the selection process more intuitive. To extract isosurfaces from volume data representing objects with branching structures, surface growing algorithms have been developed. In (Hang et Ma, 2003) and (Liu et Peng, 2015) the trivial choice of the isovalue for the isosurface extraction is replaced by region growth method.

For 3D reconstruction, most biological data require a local adjustment of the isovalue due to the variations in the boundary value given by the material. This becomes necessary for the segmentation of anatomical branches of high fractal dimension. This problem is addressed by Matyas (Matyas et al., 2005), the authors propose an automatic approach of locally varied isovalue. The resulting surface is called metasurface. The extraction of the metasurface is based on the identification of the structural information and the segment detection, which allows the local determination of the isovalue and local isosurface extraction. The proposed approach blends the contours corresponding to the different isovalues. This method is sensitive to the noise and even can detect the segment with small size. Glanznig and authors (M. Glanznig et al., 2009) present an adaptive Marching Cubes algorithm, where they propose to use an isovalue for each cube vertex (an isovalue field) instead of defining a global isovalue for all vertices. This adaptation allowed to correct the noises, contrast derivatives, local variations density and other artifacts. Surfaces are extracted, which are a continuous blend between various isosurfaces. Sudanthi and loanna (Wijewickrema and Ioannou 2013) propose an adaptive Marching Cubes algorithm for the simulation of material removal from segmented volume data. This method is a modification of the original marching cubes algorithm to make it more suitable for use in simulation applications that contain material removal tasks. This method introduces the use of an iso-interval in place of the traditional isovalue threshold. A further advantage of using iso-intervals is that multiple segments can be rendered with the same visual properties while maintaining distinct haptic properties. The proposed method is well suited for applications involving a real-time visualization and modification of segmented volume data. The methods mentioned above have been proposed to detect more than one isovalue used to display multiple isosurfaces but not to adjust or automatically determine isovalue. Other works had the objective of automating the estimation of isovalue, as (Bajaj et al., 1997) which extract isovalue automatically by examining the gradients of the volumetric scalar field and choosing the isovalue as the maximum of a weighted gradient spectrum. Weber and al (Weber et al., 2002) present a method for the detection and utilization of critical isovalues for the exploration of trivariate scalar fields by considering the topological properties of a scalar field. When using critical isovalues in volume data exploration, an extracted isosurface triangulation must have a correct topology. Navab and al (Navab and al., 2015) propose a new method that simultaneously identifies the isovalue using hierarchical clustering. Preim and Botha (Preim and Botha 2014) propose to use histogram as a support for isovalue selection. This solution proposes to choose a peak or a local maximum in the histogram as isovalue but this solution does not ensure that all the structures are in the isosurface. For this, the author proposes to extend a value slightly below the peak. This solution is also interactive. (Pekar,et al, 2001) suggested use discrete isosurface statistics to supplement histograms for detecting significant isovalues. In addition, these authors showed how to compute the Laplacian weighted histogram to find isovalues at which significant boundary effects occurred. Given the discrete nature of the Laplacian computation described, the effect of this is to convolve the image with a small interpolation kernel, then compute the histogram. (Tenginakai, et al, 2001) used multi-dimensional histograms based on discrete computations of local higher-order moments, to identify significant isovalues.

It is obvious that the histogram is a powerful tool for 
determining the appropriate isovalue, especially, if we use a good method to select it. For that, we propose to estimate the isovalue in an automatic way based on the discriminating analysis of the histogram.

\section{Background}

\subsection{Isosurface and Isovalue}

An isosurface is the set of points on the surface that have the same weight (intensity, for example). It is a surface for which a given value is always the same (isovalue).

An isosurface is a function $\mathrm{f}$ such that every point $(x, y, z)$ $\in R^{3}$,

$$
f(x, y, z)=c
$$

Where $c$ is a constant.

The choice of $c$ (isovalue) allows the user to select a particular object in the volume without using all the voxels of the volume set. This choice can be made manually or automatically (Vinicius and Pedrini 2013).

\subsection{Marching Cubes}

In order to represent a volume, the space is divided into elementary cubes of identical size cells and processes each of them separately (Preim and Botha 2014).

We realize in the following binary segmentation in order to determine all the pixels which are belonging to the volume to be reconstructed by defining an isovalue beforehand.

The Marching Cubes algorithm considers the cube as a volume element, consisting of 8 vertices and 12 edges (Chernicov and Xu 2013).

The possible triangulations of a cell are stored in a case lookup table. There are 256 possible cases, which can be reduced by topological and rotational symmetry to 15 cases.

Then, we create an index for each case, based on the state of the vertex.

$$
\begin{cases}1 & \text { ifintensity } \geq \text { isovalue } \\ 0 & \text { ifintensity }<\text { isovalue }\end{cases}
$$

After retrieving the triangles of a case from the lookup table, the positions where the triangle vertices lie on the cell edges have to be calculated. This is done with linear interpolation.

This index is used to determine the edges intersected by the surface and to interpolate the surface intersection along the edges by linear interpolation.

The Marching Cubes algorithm calculates the normals for each cube vertex and that will be used for the volume rendering:

$$
\begin{aligned}
G_{x}(i, j, k) & =\frac{D(i+1, j, k)-D(i-1, j, k)}{\Delta_{x}} \\
G_{y}(i, j, k) & =\frac{D(i, j+1, k)-D(i, j-1, k)}{\Delta_{y}} \\
G_{z}(i, j, k) & =\frac{D(i, j, k+1)-D(i, j, k-1)}{\Delta_{z}}
\end{aligned}
$$

\subsection{Automatic Marching Cubes}

In order to automate the isovalue selection, we suggest carrying out this step in an automatic way through resorting to automatic thresholding methods. Indeed, these methods are the most common techniques for extracting objects from the background of the image. Their advantages are their ease of implementation and their efficiency in real-time systems. Moreover, these techniques are based on the study of the gray levels distribution of the image pixels (study of the dynamics).

\subsection{A standard DICOM (Digital Imaging and Commu- nication in Medicine)}

The massive daily production of medical images cannot be archived in a common JPEG format at the risk of losing data associated with the image such as patient name, type of examination, hospital, etc. The DICOM format makes it possible to make each image produced unique and to associate it with specific information. This has the consequence of producing autonomous images insofar as it is always possible to formally identify their origins in case of loss, renaming or reproduction. The format is of variable size. It contains bond information and other optional. Each DICOM image must contain several types of unique identification numbers UID (Unique Identifier) automatically generated by machines. There cannot be two identical UID to designate different information and this, whatever the machine is and its location. This uniqueness is necessary not only for medical reasons, but also to allow the formation and management of databases (Chabriais. J., Gibaud. B, 2004, K. Sudharani et al., 2014).

\subsection{Automatic Isovalue Estimation}

We propose to use automatic thresholding methods that have shown their performance in the selection of threshold.

Isovalue (threshold) can be selected interactively by a user of an interactive package, but for image analysis processes that must run automatically. Then we would like to be able to compute the isovalue automatically. The basis for choosing a threshold is the histogram of the gray-ton images.

\subsection{Isovalue Calculation using Minimized the Interclass Variance Method (Otsu's Algorithm)}

Otsu's algorithm is a simple and popular thresholding method for image segmentation, it was proposed in 1967. It is also called maximum variance between clusters. Image histogram is the basis and maximum variance 

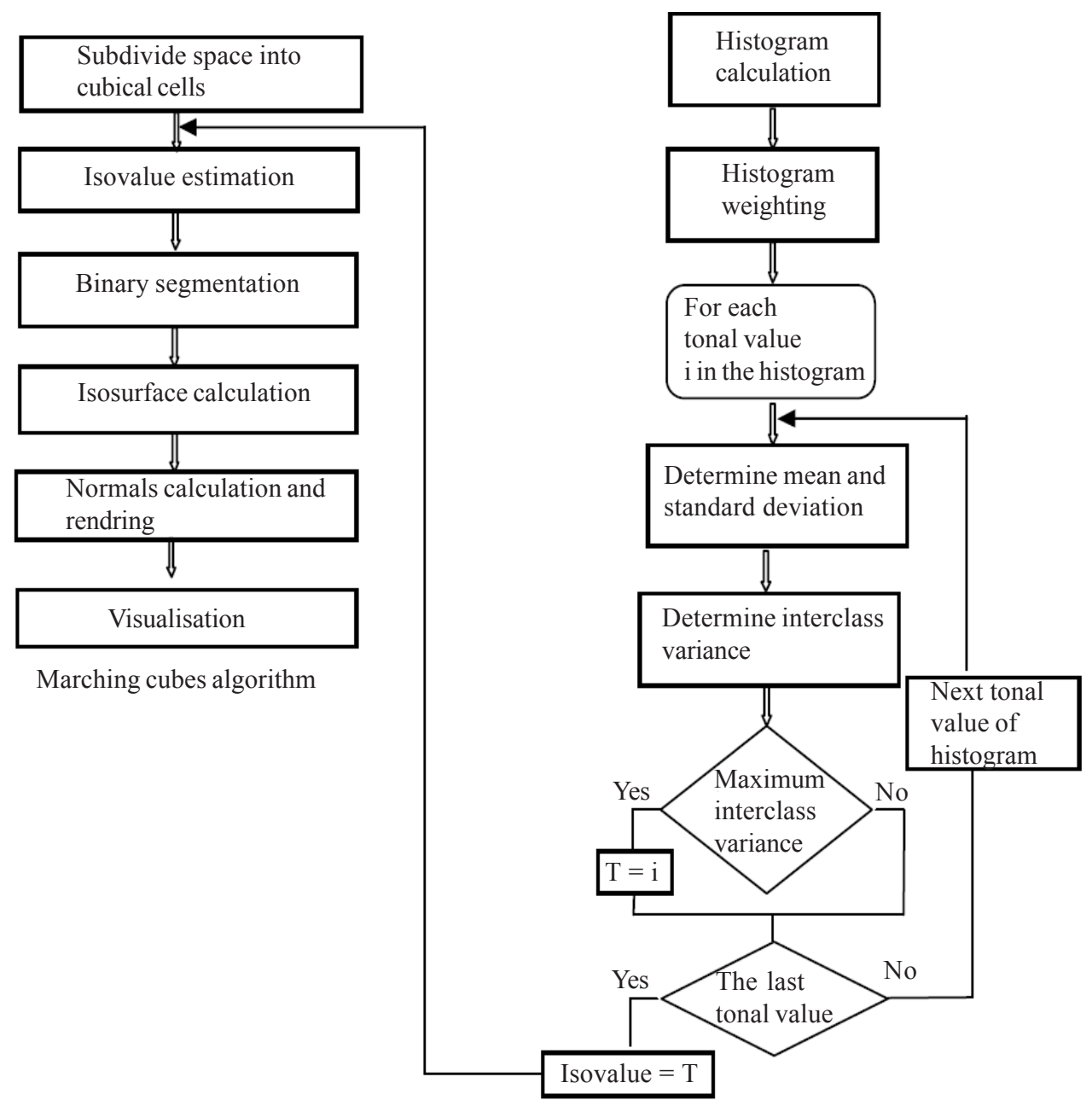

Automatic isovalue estimation

Figure 1. Proposed method diagram
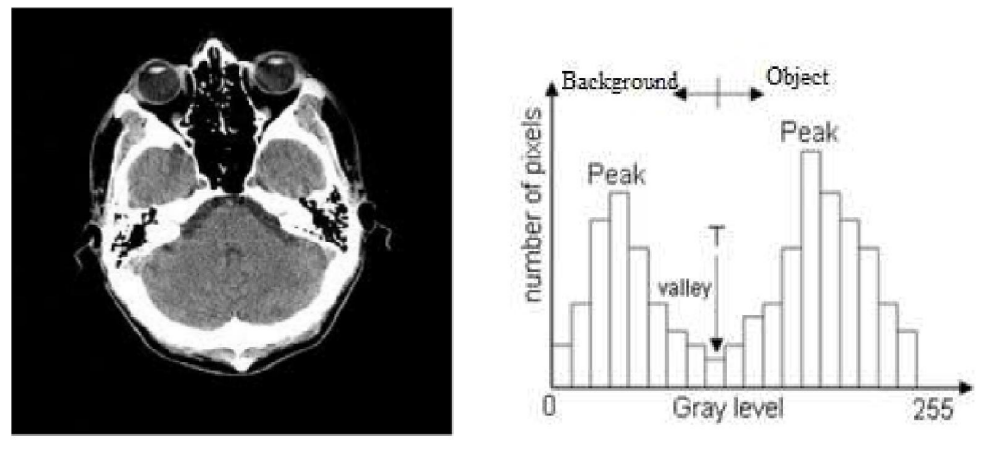

Figure 2. Original image (a) and their corresponding histogram (b) (Moallem and Razmjooy, 2012)

between target and background as the selection criteria. It uses discriminate analysis to divide foreground and background by maximizing the discriminate measure. The threshold selected by Otsu's algorithm is very ideal and is relatively good in performance in all cases (Shah and Shah, 2012).

The algorithm divides the image histogram into two classes and tries to minimize the weighted interclass variance $\sigma_{w}{ }^{2}(t)$ defined the expression (Liu, 2012, Balarini and
Nesmachnow 2016).

$$
\sigma_{w}^{2}(t)=q_{1}(t) \sigma_{1}^{2}(t)+q_{2}(t) \sigma_{2}^{2}(t)
$$

While $t$ is a value of threshold between 0 and 255 .

A probability function $P$ is obtained for every pixel value. First, the histogram distribution for the image is computed, and then normalization which is performed in order to guarantee it follows a probability distribution. After that, 
the pixel values are divided into two classes $C_{1}$ and $C_{2}$ by a threshold $t$, using the class probability functions $q 1(t)$ and $q 2(t)$ :

$$
q_{1}(t)=\sum_{i=1}^{t} p(i) q_{2}(t)-\sum_{i=t+1}^{I} p(i)
$$

Class $C_{1}$ represents those pixels with intensity levels in $[1, t]$, and class $C_{2}$ represents those pixels with levels in the interval $[t+1, I]$, where $I$ is the largest pixel value (typically 255). Then, the means for class $C_{1}, \mu_{1}(t)$, and class $C_{2}, \mu_{2}(t)$ are obtained:

$$
\mu_{1}(t)=\sum_{i=1}^{t} \frac{i p(i)}{q_{1}(t)} \mu_{2}(t)=\sum_{i=t+1}^{I} \frac{i p(i)}{q_{2}(t)}
$$

After that, the variances for class $C_{1}, \sigma_{1}^{2}(t)$, and class $C_{2}$, $\sigma_{2}^{2}(t)$ are computed:

$$
\begin{aligned}
& \sigma_{1}^{2}(t)=\sum_{i=1}^{t}\left[i-\mu_{1}(t)\right]^{2} \frac{p(i)}{q_{1}} \\
& \mu_{1}(t)=\sum_{i=t}^{t} \frac{i p(i)}{q_{1}(t)} \mu_{2}(t)=\sum_{i=t+1}^{I} \frac{i p(i)}{q_{2}(t)}
\end{aligned}
$$

$\sigma_{1}^{2}(t)$ and $\sigma_{2}^{2}(t)$ are the weighted within-class variance for $C 1$ and $C 2$, respectively that Otsu's algorithm tries to minimize.

\section{Results, Validation and Discussion}

For the validation of the proposed method, we use three databases, each one consisting of a series of public DICOM images axial of CT-head, ankle, pelvic and foot (resolution: $512 \times 512$, samples per pixel: 1 , pixel spacing: 1.00/1.00, slice thickness: 1).

\subsection{Automatic Selection of Isovalue}

The first step of our method consists in calculating the automatic isovalue. This step is achieved by using the interclass variance maximization algorithm of Otsu. However, we have to manipulate not a single image but a series of images, each has its own isovalue. We ended up with a set of isovalues. In order to choose the optimal isovalue, we have made a survey to evaluate the thresholding quality of several dataset images considering the means, the minimum and maximum of this set of values. The evaluation is carried out by the Region NonUniformity measure (NU) (Sezgin and Sankur, 2004) which does not require ground-truth information, is defined as:

$$
N U=\frac{\left|F_{T}\right| \sigma_{f}^{2}}{\left|F_{T}+B_{T}\right| \sigma^{2}}
$$

Where $\sigma^{2}$ represents the variance of the whole image, $\sigma_{f}^{2}$ represents the foreground variance. It is expected that a well-segmented image will have non-uniformity measure close to 0 while the worst case of $N U=1$ corresponds to an image for which background and foreground are indistinguishable up to second order moments.

The error $N U$ is calculated on a sample of images randomly draw from the three used database, the following table illustrates the results obtained:

\begin{tabular}{|l|l|l|l|}
\hline NU & Sample1 & Sample2 & Sample3 \\
\hline Max & 0.009327 & 0.019632 & 0.000131 \\
\hline Min & 0.033806 & 0.107850 & 0.020864 \\
\hline Means & 0.011184 & 0.047576 & 0.003318 \\
\hline
\end{tabular}

Table 1. Thresholding evaluation

We have observed that the best performing thresholding is obtained by considering the maximum value of all thresholds and this, for the images of the three datasets.

In order to highlight the proposed method, we proceeded for a beginning to 3D reconstruction by Marching Cubes algorithm by initialization the isovalue in an interactive way (figure 5). Second, we performed a 3D reconstruction using the proposed (automatic Marching Cubes) algorithm and we analysed and compared the volumes obtained. We noticed that in the case of choosing an interactive isovalue lower than the automatic isovalue, one finds the presence of objects which are not a part of the reconstructed volume. This is justified by the fact that vertices which have a radiometric value close to that of the isosurface were considered belonging to the volume during the Marching Cubes 3D reconstruction algorithm.

\subsection{Construction Time}

One important aspect when comparing two methods is the time for generating the iso-surface. Table 2 presents some experimental results.

\begin{tabular}{|l|c|l|c|}
\hline \multicolumn{2}{|c|}{ Images } & \multicolumn{2}{c|}{ Time (seconds) } \\
\hline Name & Size & $\begin{array}{l}\text { Automatic } \\
\text { isovalue }\end{array}$ & $\begin{array}{c}\text { Interactive } \\
\text { isovalue }\end{array}$ \\
\hline CT-head & $512 \times 512 \times 350$ & 6.95 & 7.70 \\
\hline Ankle & $512 \times 512 \times 246$ & 7.01 & 8.06 \\
\hline Foot & $512 \times 512 \times 252$ & 10.40 & 10.60 \\
\hline
\end{tabular}

Table 2. Construction time

We observe that the automatic Marching Cubes algorithm always spend less time than the interactive one.

\subsection{Volumes Quality}

We also compared the quality of the volumes obtained by the proposed method and the interactive marching cubes. For this we used the Side-by-side eye-sight comparison cited by Xuebin Xu (Xuebin, 2003). 
The results shown in the following figures are obtained by the 3D reconstruction of 350 images of CT-head.

The analysis of the reconstructed volumes revealed that the errors of reconstruction are accentuated when the value of the interactive isovalue is chosen smaller and smaller than that of the automatic isovalue. This is explained by the increase in the number of vertices that do not belong to the volume and that are considered in the volume by the Marching Cubes algorithm.
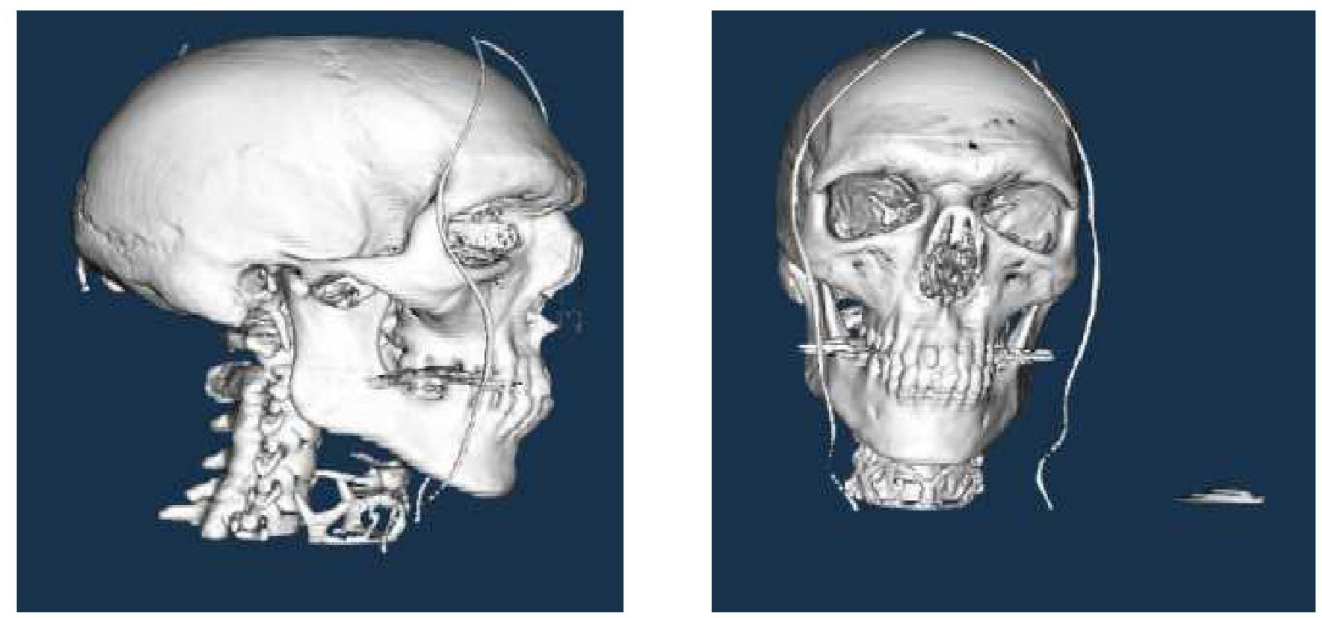

Figure 3. 3D reconstruction for automatic isovalue (isovalue $=150)$

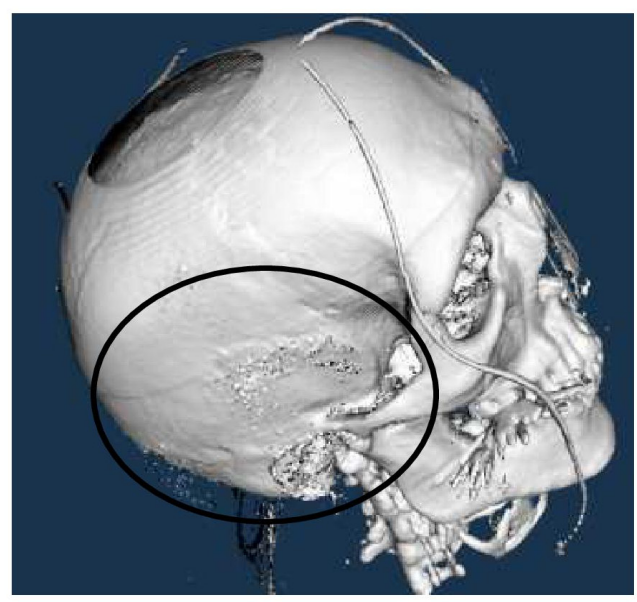

Interactive isovalue (120)

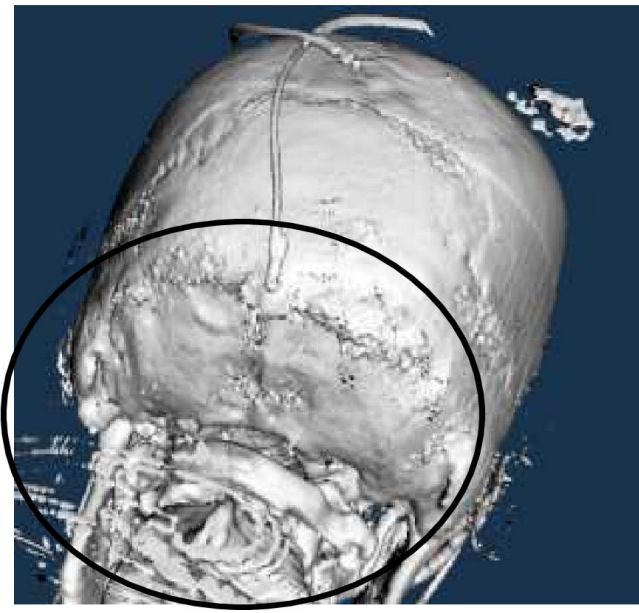

Interactive isovalue (120)

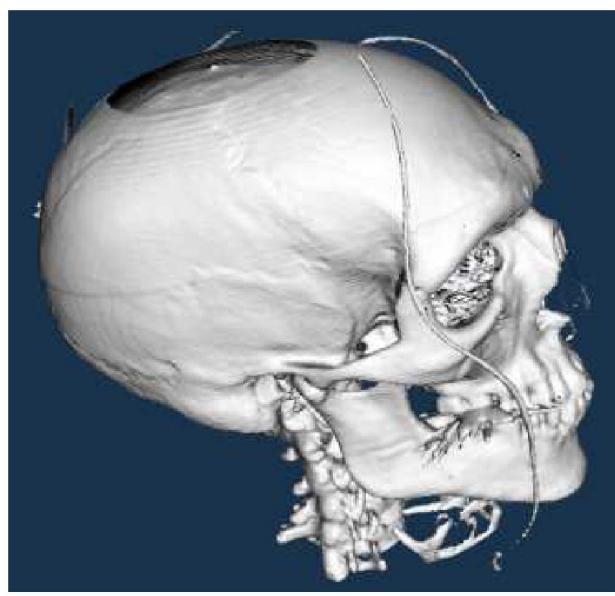

Automatic isovalue (150)

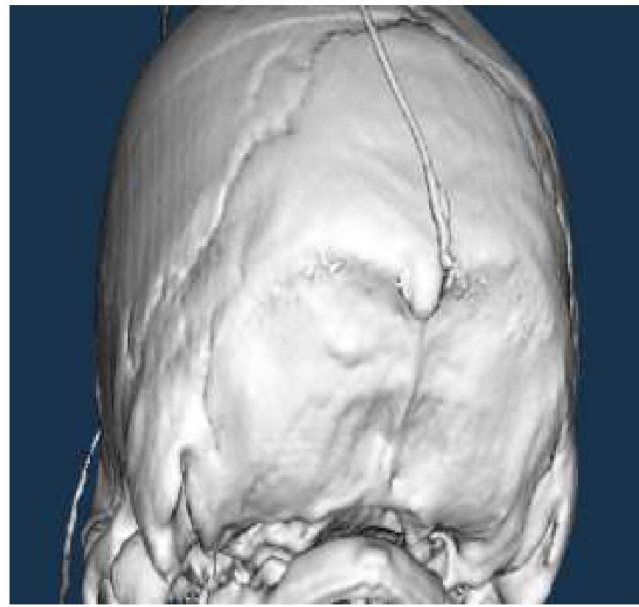

Automatic isovalue (150)

Figure 4. Noise more present on the volume reconstructed by the interactive isovalue that on the volume reconstructed by the automatic isovalue 


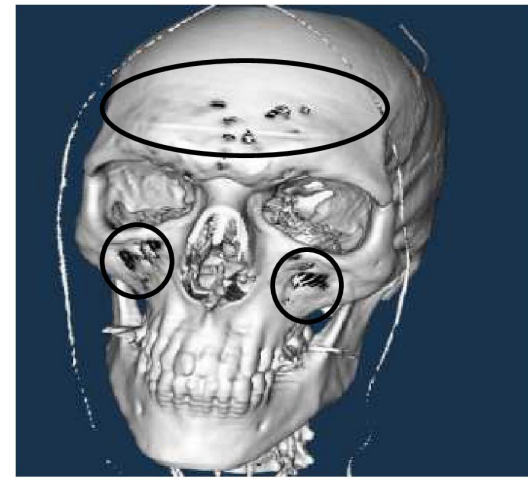

Interactive isovalue $=200$

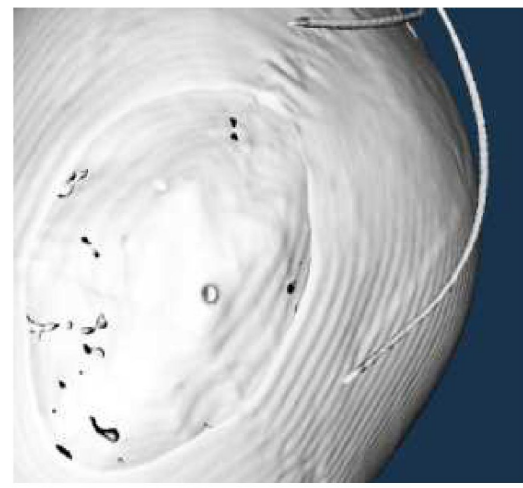

Interactive isovalue $=245$

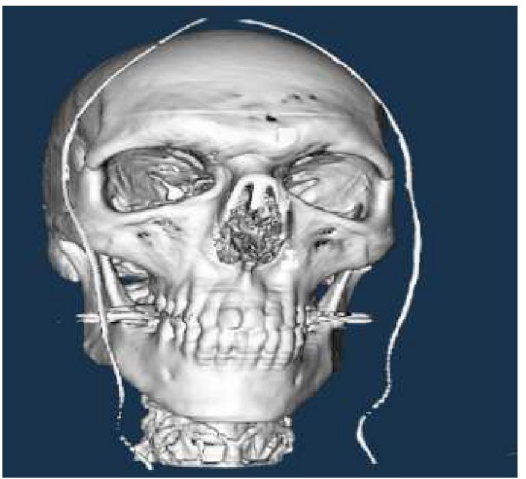

Automatic isovalue $=150$

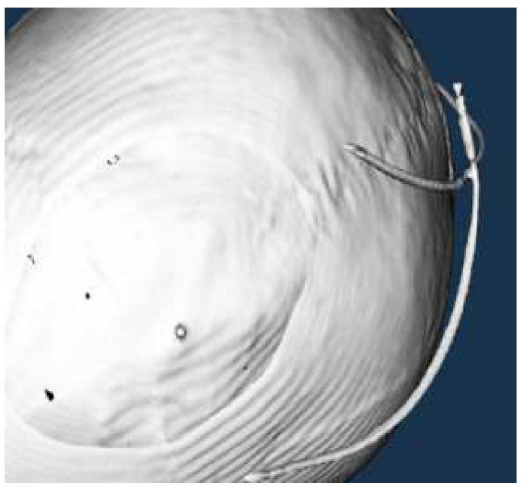

Automatic isovalue $=150$

Figure 5. Presence of the holes on the volume reconstructed by the interactive isovalue superior to the automatic isovalue

In the case of the choosing an interactive isovalue greater than the automatic isovalue, we note the presence of the holes while these defaults are less present in the volumes reconstructed from the automatic isovalue (figure 5).

We also found that the holes are more present in the case of the choice of interactive isovalues more and more superior to the automatic isovalue. This explains that an upper interactive isovalue induced to the classification of the vertices, in the volume, as out of the volume by the Marching Cubes algorithm.

Our volumes were obtained from a single reconstruction while with a preliminary knowledge of the image, and after having realized several reconstructions, we could not fall on the optimal isovalue, which allows us to obtain higher quality volumes because the probability of choosing the same isovalue calculated automatically is $1 / 256$ (0.004), which is a rather small probability.

The proposed method, therefore, allows a considerable saving of time, even with the taking into account of the calculation time of the automatic isovalue, which is of a few seconds.

It should also be noted that the volumes obtained by the automatic Marching Cubes algorithm are the same size as those obtained by the classical Marching Cubes algorithm.

We can see the same reconstruction errors in the other image datasets used for the validation of the proposed method:
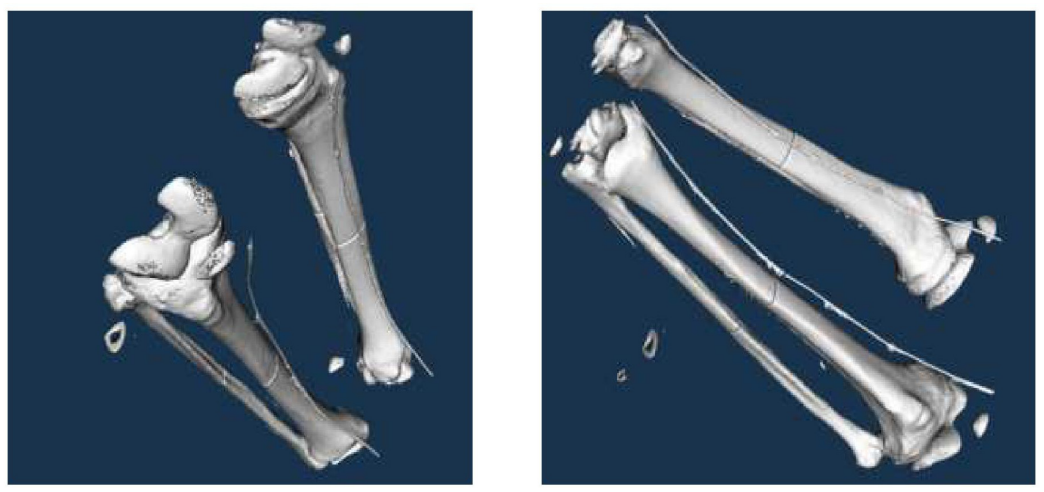

Figure 6.3D reconstruction from the automatic isovalue 


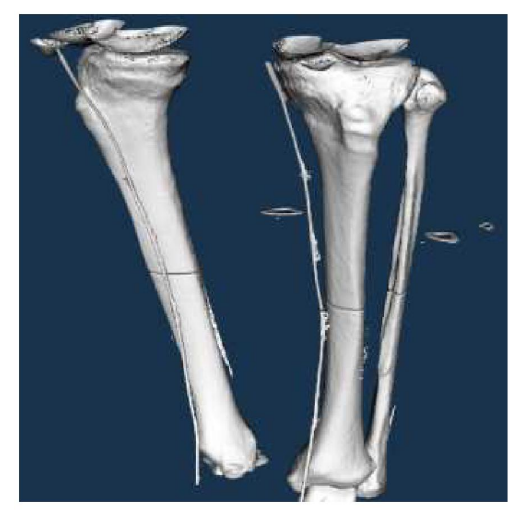

(a)

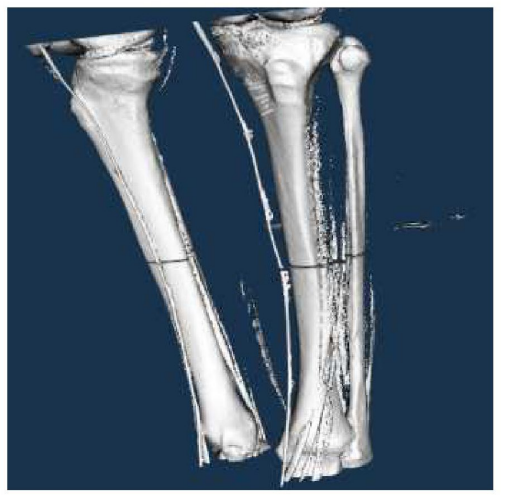

(a)

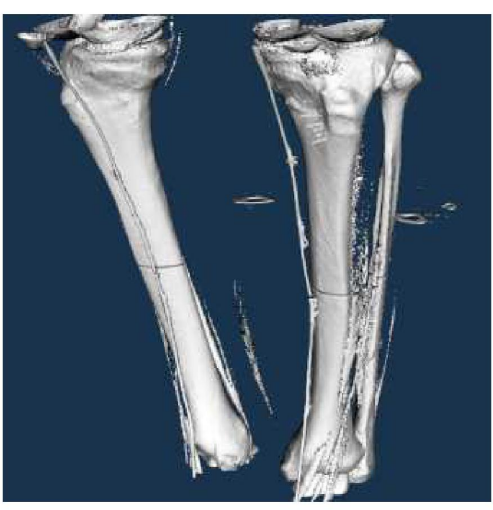

(b)

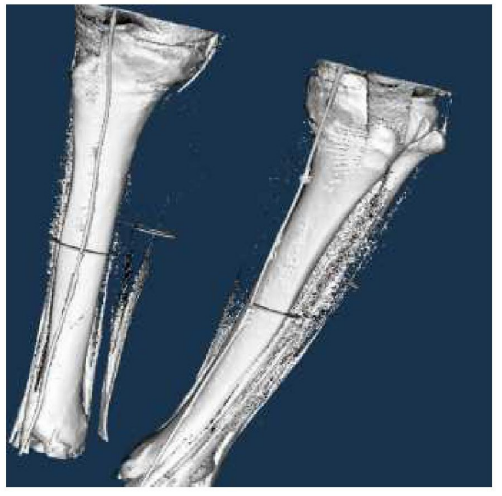

(b)

Figure 7. 3D reconstruction of dataset 2 by an automatic (a) and interactive isovalue (b, c, d) lower than the automatic isovalue (presence of noises)

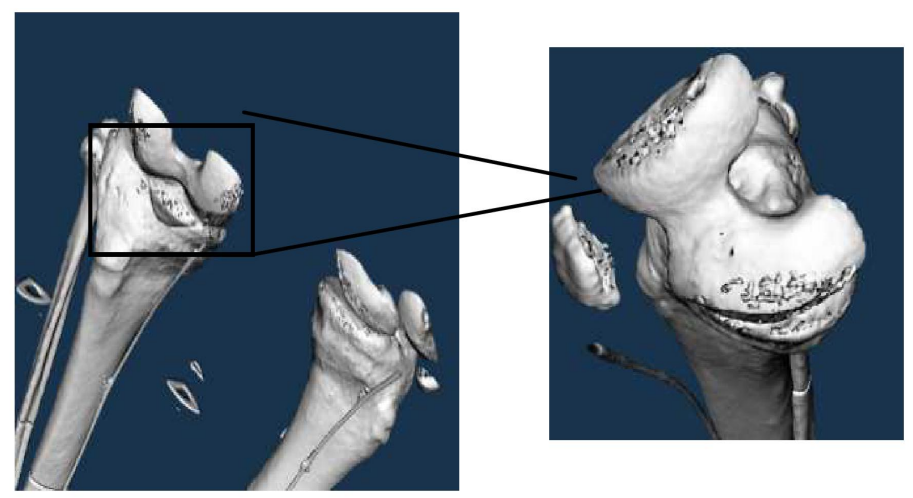

Automatic isovalue

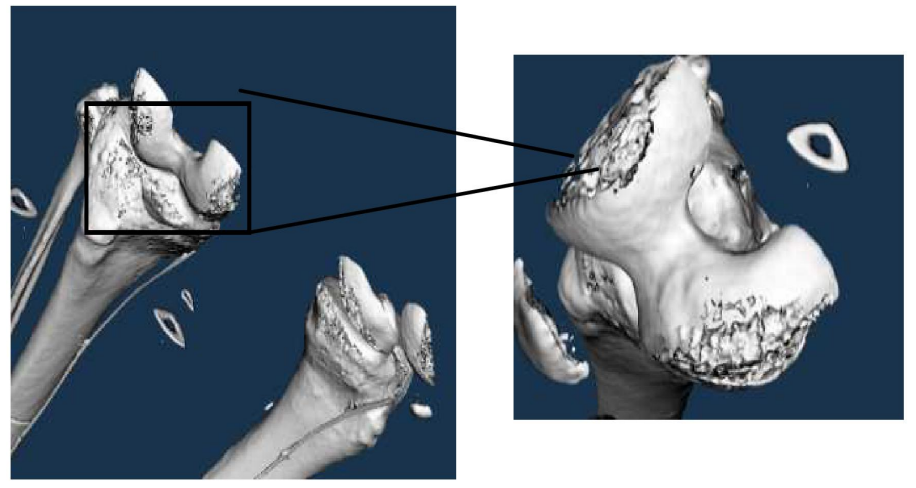

Interactive isovalue

Figure $8.3 \mathrm{D}$ reconstruction of the dataset 2 by an interactive isovalue is higher than the automatic isovalue (presence of holes) 


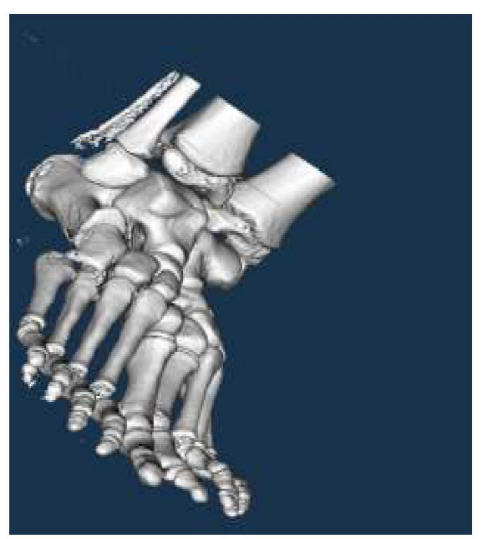

Automatic isovalue

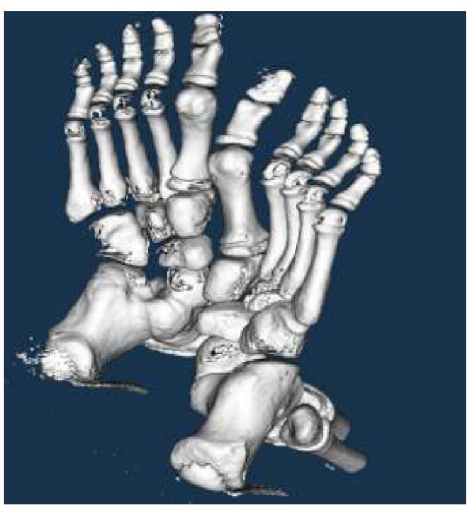

Automatic isovalue

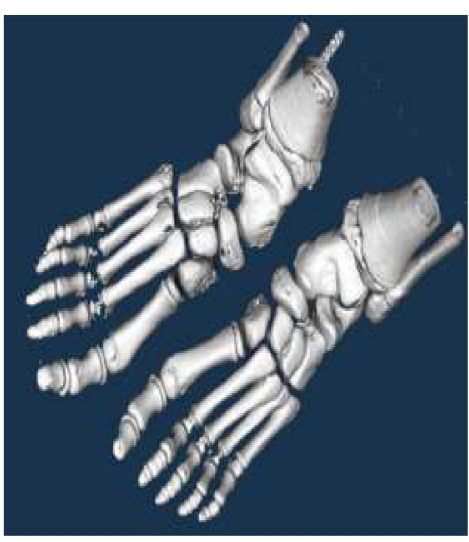

Interactive isovalue

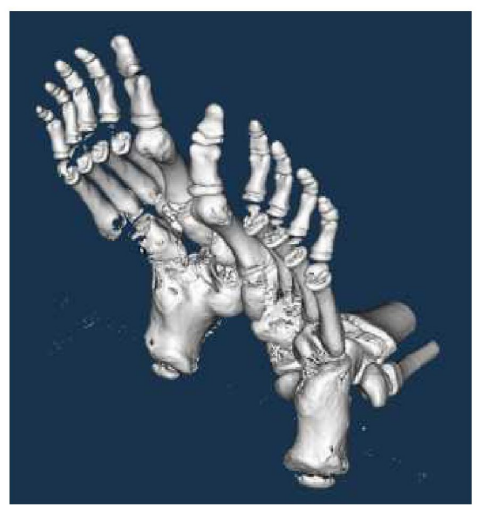

Interactive isovalue

Figure $9.3 \mathrm{D}$ reconstruction from the automatic isovalue

We noticed the presence of some holes on the reconstructed volumes in our method. This is due to the fact that the automatic isovalue chosen is the maximum value of all the isovalues obtained from the dataset images. This led to the classification of several vertices of the volume as vertices out by the automatic Marching Cubes algorithm.

This error can be raised by considering an automatic adaptive Marching Cubes algorithm, that is, considered an appropriate isovalue for each dataset image.

\section{Conclusion and Future Work}

In this paper, we have presented an automatic Marching Cubes for an improvement 3D medical image reconstruction.

The modification of the Marching Cubes algorithm is done to automate the isovalue selection phase during the isosurface extraction. This is motivated by the fact that this step is performed in an interactive way and without any orientation or taking into account the image dynamics.

The brought changes are realized, using Otsu's automatic thresholding method that is simple to implement and gives good results in the selection of an image threshold.

We have shown through the obtained results that the selection of a small value we obtain false positives: the reconstructed volume induces vertices, which are not a part of the object of interest. It is usually about a noise or the structures of another nature that have a grey level too close to that object of interest. In addition, the choice of a more or less high isovalue induces false negative. That is, some elements of the object of interest may not appear or only partially.

We also showed that the volumes obtained by the proposed method are of better quality than those obtained by the classical Marching Cubes algorithm. Thus, our volumes are obtained from a single reconstruction, which allows a considerable saving of time and that probability of choosing the same value as the automatic isovalue is very small.

As future work we would like to introduce an automatic adaptive isovalue appropriate for each dataset image and introduce the rules of probability for the vertices classification, with the aim of obtaining the best possible results.

\section{References}

[1] Bajaj, C. L., Pasucci. V., Schikore. D. R. (1997). The contour spectrum, IEEE Computer Society Press, 167173. 
[2] Balarini, J. P., Nesmachnow, S. (2016). A C++ Implementation of Otsu's Image Segmentation Method, Image Processing On Line, 155-164.

[3] Chabriais, J., Gibaud, B. (2004). DICOM, le standard pour l'imagerie médicale, EMC - Radiologie. 1 (6).

[4] Chernicov, A., Xu, J. (2013). A Computer-Assisted Proof of Correctness of a Marching Cubes Algorithm, the $22^{\text {nd }}$ International Meshing Roundtable, 505-523.

[5] Huang, R., Ma, K. (2003). Region growing based techniques for volume visualization, In: Jon Rokne, Reinhard Klein, and Wenping Wang, editors, Pacific Conference on Computer Graphics and Applications 2003, 355-363. IEEE Computer Society Press.

[6] Glanznig, M., Malik, M. M., Gröller, M. E., (2009). Locally adaptive marching cubes through iso-value variation, In: Pro of International Conference in Central Europe on Computer Graphics, Visualization and Computer Vision, 33-40.

[7] Lyness, C., Blake, E. (2001). Real Time Isosurface Browsing, AFRIGRAPII.

[8] Liu, S., Peng, J. (2015). Optimization of Reconstruction of 2D Medical Images Based on Computer 3D Reconstruction Technology, Journal of Digital Information Management, 13 (3).

[9] Liu, Y. (2012). Study on Automatic Threshold Selection Algorithm of Sensor Images, International Conference on Solid State Devices and Materials Science. Physics Procedia 25. $1769-1775$.

[10] Marks, J., Andalman, B., Beardsley, P. A., Freeman, W., Gibson, S., Hodgins, J., Kang, T., Mirtich, B., Pfister, H., Ruml, W., Ryall, K., Seims, J., Shieber, S. (1997). Design galleries: a general approach to setting parameters for computer graphics and animation, In: 24th Annual Conference on Computer graphics and interactive techniques (SIGGRAPH 1997), 389-400.

[11] Matyas, N. M., Linseny, L., Hamann, B. (2012). Metasurfaces: Contouring with Changing Isovalue, VMV 2005 Erlangen, Germany, 147-157.

[12] Moallem, P., Razmjooy, N. (2012). Optimal Threshold Computing in Automatic Image Thresholding using Adaptive Particle Swarm Optimization, Journal of Applied Research and Technology, 10, 703-712.

[13] Navab, N., Hornegger, J., Wells, W. M., Frangi, A. F. (2015). Medical images computing and computer-assisted intervention, In: MICCAI, 18th International Conf Munich, Germany, Proceedings, Part III.
[14] Newman, T. S., Yi, H. (2006). A survey of the marching cubes algorithm, Computers \& Graphics, 30, 854879.

[15] Pekar, V., Wiemker, R., Hempel, D. (2001). Fast Detection of Meaningful Isosurfaces for Volume Data Visualization, In: Proceedings of Visualization 2001, 223230.

[16] Preim, B., Botha, C. (2014). Visual Computing For Medicine Theory, Algorithms, and Applications, Second Edition. Elsevier Inc.

[17] Shah, B., Shah, A. P. S. (2012). Implementation of Image Segmentation on Digital Images Using Modified Otsu Algorithm, International Journal of Innovative Technology and Exploring Engineering (IJITEE), 1 (1).

[18] Sezgin, M., Sankur, B. (2004). Survey over image thresholding techniques and quantitative performance evaluation, Journal of Electronic Imaging 13 (1) 146165.

[19] Sudharani, K., Sudharani, K., Reddy, Ravindra., R., Sarma, T. C., Prasad, Satya., K. (2014). 3D DICOM images reconstruction using LAB VIEW, International Journal of Electrical, Electronics and Data Communication, 2(1).

[20] Tenginakai, S., Lee, J., Machiraju, R. (2001). Salient Iso-Surface Detection with Model-Independent Statistical Signatures. In: Proceedings of Visualization 2001, 231238.

[21] Vinicius, M., Cirne, M., Pedrini, H. (2013), Marching cubes technique for volumetric visualization accelerated with graphics processing units, Journal of The Brazilian Computer Society, 223-233.

[22] Weber, G. H., Scheuermann, G., Hagen, H., Hamann, B. (2002). Exploring Scalar Field using Critical Isovalues, IEEE Visualization, 171-178.

[23] Wijewickrema, S., loannou, I. (2013). Adaptation of Marching Cubes for the Simulation of Material Removal from Segmented Volume Data, In: $26^{\text {th }}$ International Symposium on Computer-Based Medical Systems (CBMS, 2013).

[24] William, E. L., Harvey, E. C. (1987). Marching Cubes: A high resolution $3 \mathrm{D}$ surface construction algorithm, $14^{\text {th }}$ annual conference on Computer graphics and interactive techniques (SIGGRAPH 87), 21, 163-169.

[25] Xuebin, X. (2003). A Survey in Volumetric Comparison Algorithms Suitable For the Research of Correlative Visualization, MEDIANET select survey series, Department of computer science Kent State University. 